Research Article

\title{
Based on Temporal and Spatial Traffic Characteristics for Energy-Efficient Wi-Fi Network
}

\author{
Zhigang Li $\mathbb{D},{ }^{1}$ Xin Wang $\mathbb{D}^{1},{ }^{1}$ Xinan Yue $\mathbb{D}^{1},{ }^{1}$ Dongyao Zou, ${ }^{1}$ Jun Li $\mathbb{D},{ }^{2}$ and Lina $\mathrm{Si} \mathbb{D}^{1}$ \\ ${ }^{1}$ College of Computer and Communication Engineering, Zhengzhou University of Light Industry, Zhengzhou 450002, China \\ ${ }^{2}$ Henan Co. Ltd., China Mobile Communications Group, Zhengzhou 450008, China \\ Correspondence should be addressed to Zhigang Li; lizg.cn@hotmail.com
}

Received 21 July 2021; Revised 15 September 2021; Accepted 8 October 2021; Published 23 October 2021

Academic Editor: Jun Li

Copyright (c) 2021 Zhigang Li et al. This is an open access article distributed under the Creative Commons Attribution License, which permits unrestricted use, distribution, and reproduction in any medium, provided the original work is properly cited.

With the rapid development of information and communication technology (ICT) industry, the energy consumption caused by the development of mobile communications is increasing exponentially. Huge energy consumption and increasing environmental awareness prompt people to study the dynamic distribution of users and traffic in Wi-Fi networks in order to design an energyefficient mechanism for Wi-Fi networks. Based on real data, this paper explores the spatial and temporal characteristics of human mobility and data traffic distribution. The results of access point (AP) traffic analysis show a tidal effect and obvious periodicity in time and space dimension, which can be used for the dynamic design of AP sleep or shutdown strategy. At the same time, we design a new service area (SA) zooming and AP cooperation mode. Through the simulation experiment, we find that the energy consumption can be reduced by about $35 \%$ and the communication service quality can be guaranteed.

\section{Introduction}

Over the past few years, there have been significant technological advances in the field of ICT, with an explosion of data-intensive applications in communication networks. At the same time, the number of Wi-Fi access points has grown exponentially with the use of mobile devices such as smartphones, tablets, and laptops, because of the need to provide users with high data rates to access the Internet anytime and anywhere. The huge data traffic comes at the cost of an increasing carbon footprint and huge energy consumption. Relevant studies show that energy consumption in the ICT industry accounts for $2 \%$ to $10 \%$ of the world's total energy consumption, while more than $55 \%$ of energy consumption in communication systems occurs in access networks [1]. The energy efficiency of infrastructures in information and communications is a major concern of industry, government, and science. In addition, higher energy consumption means higher capex and operating expenses for network operators. Therefore, reducing energy consumption in ICT is a major research challenge, which is also extremely important to address from an ecological and economic point of view.
In the work [2], by analysing the mobility characteristics of people, they found that the fluctuation of the number of people in a region exists in many places, such as commercial areas, office areas, and residential areas, and there are great differences between day and night. They studied human mobility patterns and found that most people's activities in their daily lives have obvious periodicity and tidal characteristics [3]. As the main body of traffic generation, human's temporal and spatial movement will inevitably lead to nonuniformity and uncertainty of traffic. In Wi-Fi network, therefore, network resources are usually provided according to peak capacity requirements and kept in nondowntime operation state to ensure that users can access Wi-Fi network at any time and in any place. When the network coverage area is under low load, the resources of AP cannot be fully utilized, resulting in a large number of SAs in the state of over-coverage for a long time, and a large number of resources are wasted. In particular, emerging data and video broadcasting services tend to tolerate a certain amount of delay, and the network does not always operate at peak traffic. In addition, the rapid popularity of Internet of Things applications can bring a large number of medium and low business volumes to the network. The business requests are 
small but very frequent, which actually consume a lot of energy of AP.

By collecting and measuring the service traffic of Wi-Fi network system, the distribution characteristics of the service traffic can be obtained. Traffic in Wi-Fi network is not uniformly distributed in time domain and space. If people's mobility characteristics are combined with traffic fluctuations, we can find more information to guide the energy efficiency design of Wi-Fi network. Based on this idea, this paper discusses the reasons for the imbalance and fluctuation of traffic in Wi-Fi network, which should be mainly attributed to the mobility of human beings in time and space, such as the user's day and night mobility. Many previous studies $[4,5]$ have shown that human trajectories exhibit a high degree of temporal and spatial regularity and indicate that human mobility has an average predictability of $93 \%$, which is a very high value rooted in the inherent regularity of human mobility. This inherent similarity in mobility patterns could have implications for all phenomena driven by human mobility, such as in the energy-efficient design of Wi-Fi networks.

By analysing the real dataset of Wi-Fi network collected by Dartmouth College [6], we found that the temporal and spatial characteristics of human mobility are highly consistent with the temporal and spatial characteristics of data traffic [7]. In addition, we need to explore the privacypreserving techniques in location-based services in the following work [8]. Based on these characteristics, we can explore more feasible energy-efficient design methods. The main contribution of this paper is to prove the tidal effect and obvious periodicity of Wi-Fi network traffic in time and space through the analysis of real Wi-Fi network datasets and to guide the energy-efficient design of Wi-Fi networks. We adopted SA zooming and AP Cooperation mechanism to reduce the network energy consumption. Through simulation experiments, we found that we saved about $35 \%$ of the energy consumption.

The rest of this paper is organized as follows: in the second section, we analyse the current work related to energy-efficient design of wireless networks. In the third section, we explore the spatiotemporal traffic characteristics of Wi-Fi networks. The fourth part describes the Wi-Fi network energy-efficient design scheme and energy-efficient effect analysis. We summarize this paper in the fifth part.

\section{Related Works}

In recent years, a great deal of research has emerged on the energy-efficient aspect of ICT. In the idle state of the equipment, the overall energy consumption of the equipment will increase. The sleep setting will be based on the state of the equipment to fundamentally reduce the overall energy consumption. The dormant period and working period of the wireless network cannot be freely converted according to the production and operation requirements, resulting in the relatively low control and management ability of the wireless network equipment for its own dormant state. This paper aims to effectively reduce the energy consumption of the wireless network itself, realize the dynamic monitoring and management of the dormant period, and then fundamentally improve the overall energy-efficient level of the wireless transmission sensor. On this basis, the intelligent dynamic control of equipment can be completed by setting the priority level management of equipment. In addition, the total heat dissipation of the chip in the internal structure of the equipment is the main technical source of energy consumption generated by the equipment. The new communication control can effectively manage and master the equipment state in the nonworking environment and effectively prevent the unnecessary energy loss of the equipment in the nonworking state.

In this paper, we mainly analyse and discuss the energyefficient research of wireless access point equipment in wireless network. The work [9] proposes the algorithm for energy-efficient operations in systems based on IEEE 802.11 WAPs. Increasing the energy efficiency and improvement in providing DiffServ operations strongly depend on practical implementation and traffic management rules. By managing RF RSSI coverage maps, it is possible to select the optimal beam-formed antenna radiation pattern at a given time interval. Sharma et al. discussed the use of actor-critical reinforcement learning to switch APs according to traffic load changes. The simulation results of $20 \mathrm{APs}$ in a Wi-Fi network show that the proposed framework can greatly save energy consumption compared with the case without considering AP switching [10]. Han et al. [11] designed two implicit mechanisms to play the role of the NAV. Their mechanisms predict the duration of an imminent LTE transmission by predicting the frame's traffic class. The prediction is based on the elapsed idle slots between successive transmissions and the transmission history. They show that their methods achieve significant energy efficiency without stifling transmission opportunities. In addition, Dziedzic et al. [12] have provided a proof-of-concept, both theoretically and experimentally, of a purely energy detection based on algorithm that can be used by a LTE-U BS to determine whether there is one or two Wi-Fi APs on the air so that the duty cycle can be adjusted accordingly. Sathya et al. [13] implement and validate the proposed ML-based approach and demonstrate that there exist distinct patterns between the energy distributions between one and many $\mathrm{Wi}$ Fi AP transmissions. This method has higher accuracy.

The uneven distribution in time and space in the state of peak traffic in a particular residential area makes the overcoverage of a large number of residential areas for a long period of time, thus bringing more energy consumption and operating costs [14]. Therefore, a large number of energyefficient cellular network design schemes arises at the historic moment. Related work includes [15-17], where they introduced the use of dynamic BS operations to reduce energy consumption, allowing the system to completely shut down underutilized BSs during periods of low traffic and transfer their load to adjacent BSs. In the work [18], the author proposes a cell activation mechanism that enables BSS to be repelled and activated according to the traffic demand so that the effective BSS density can be expanded when the traffic fluctuates. They propose a cellular amplification mechanism, and the results show that power consumption can reduce the traffic demand by turning off some 
BSS and extending the low coverage of BSS in other periods $[19,20]$. Liu et al. proposed the Aligner algorithm [21]. Compared with the energy consumption for idle waiting, the slight energy consumption for overhearing an ongoing data frame is cost-effective.

In addition, the numerical analysis in the work [22] shows that the unloading strategy based on cell scope expansion greatly reduces the traffic of the macro cell, while bringing a small increase in the traffic of the outer cell. Lahiry [23] proposed a low-cost antenna array system and an adaptive cell density technology for energy-efficient longterm evolution of mobile wireless networks. In the work [24], a programmable phase-binary coding metasurface, working at $2.45 \mathrm{GHz}$, has been designed, and an efficient optimization algorithm of online adjusting the spatial intensity distribution of Wi-Fi signals has been developed. Selected experimental results have been provided to verify the proposed strategy in the dynamic energy allocation of Wi-Fi signals. He et al. [25] proposed a local routing adjustment algorithm. After a new path is found, the local routing adjustment algorithm can be performed to make local adjustments to the selected path to further reduce wireless interference. In addition, a deep learning-based prediction method is proposed [26], capturing the dynamic time-varying heat value of the hot zones based on the analysis of the real-world private car trajectory data. In the meantime, enhance the mobile-edge computing energy efficiency with end users' quality-of-experience guaranteed.

All the above methods dynamically deploy wireless access points according to actual traffic demand or low traffic cycle. However, they only explain the phenomenon of uneven traffic distribution, without specific analysis of the characteristics of such traffic. To design a more flexible system to reduce energy consumption strategy, we need SA traffic data over time and space fluctuation characteristics and then need to know our energy-efficient scheme design.

\section{Temporal and Spatial Traffic Characteristics Analysis}

In this paper, we use real datasets to analyse the spatial and temporal characteristics of data traffic in Wi-Fi networks. We used a dataset of wireless access points on the Dartmouth campus [6], where nearly 500 access points have been installed, covering most of the campus. The dataset was collected over a period of five years. The campus has 200 acres of area with more than 190 buildings, about 5,500 students and 1,200 teachers. They used three techniques to collect data which are syslog events, SNMP polls, and network sniffers, respectively. On the basis of the campus construction plan, the campus is divided into six main types of area: they are academic (Acad), administrative (Adm), athletic (Athl), library (Lib), social (Soc), and residential (Res), respectively. The Soc area includes public centre and dining area. We analysed the data from three of these months and found a high degree of consistency in the inherent regularity of the data traffic. Next, we will use some of the analysis results to illustrate and discuss the characteristics of the traffic.
In Figure 1, we show the temporal and spatial characteristics of the data traffic for one week. The corresponding regional axes are Acad, Adm, Lib, Athl, Soc, and Res. From what we observe in a week, all regions show the same characteristics every day, that is, the data traffic in a day generally reaches a high peak near 10 O'clock. Then, the traffic starts to taper off as the night going on, and between 2 $O$ 'clock and 5 O'clock, the data traffic is the lowest in one day. We find that the characteristics of the traffic were obviously tidal phenomena. As can be seen from the figure, the traffic of Res and Acad areas is higher in the daytime than that of other areas. It is believed that students in our campus as the main population are the cause. Students have classes in the teaching area during the day while they mostly stay in the accommodation area during the other time.

It is not difficult to find from Figure 1 that the traffic of the six areas is obviously cyclical, reaching a peak every 24 hours. In the case of the Acad area, traffic is high during the day and almost no traffic is generated at night. It is understandable that during the day, there are more users in the Acad area, and they all stay longer, so traffic naturally increases. At night, there are few users in the Acad area, so traffic is close to zero. In addition, we find a significant drop in traffic to the Acad area during the last two days of the week due to fewer classes and fewer students entering the Acad area. The traffic in the residential areas is always much higher than that in the other areas, which means there are many students who spend most of their time in the dormitories. A small number of users entering the Adm, Athl, and Lib areas and their short stay time have resulted in a consistently low traffic in these areas.

According to the above findings, we can find that the distribution of traffic in time and space is not uniform. Therefore, we can design an efficient and energy-efficient Wi-Fi network according to the spatiotemporal fluctuation characteristics of traffic, that is, design the strategy of dynamic sleep or shutdown of AP.

\section{Energy-Efficient Design and Effect Analysis}

Advanced power transmission and new power management solutions need to be designed to accommodate different traffic load variations to match energy consumption with actual performance. Therefore, we will reduce the coverage area by appropriately adjusting the transmission power of $\mathrm{AP}$, namely, the SA scaling mechanism, so as to save the energy consumption of Wi-Fi network.

4.1. SA Zooming and AP Cooperation Mechanism. The SA scaling mechanism we designed is designed according to the fluctuation of each Wi-Fi traffic, dynamically reducing the transmission power of AP or even completely turning off the power according to the traffic under each SA. When some APs choose to reduce transmission power or shut down, the corresponding traffic load is transferred to adjacent operating APs, thereby reducing the energy consumption of network operation by reducing transmission power. Because some APs have traffic well below peak times for most of the day, surviving APs can 


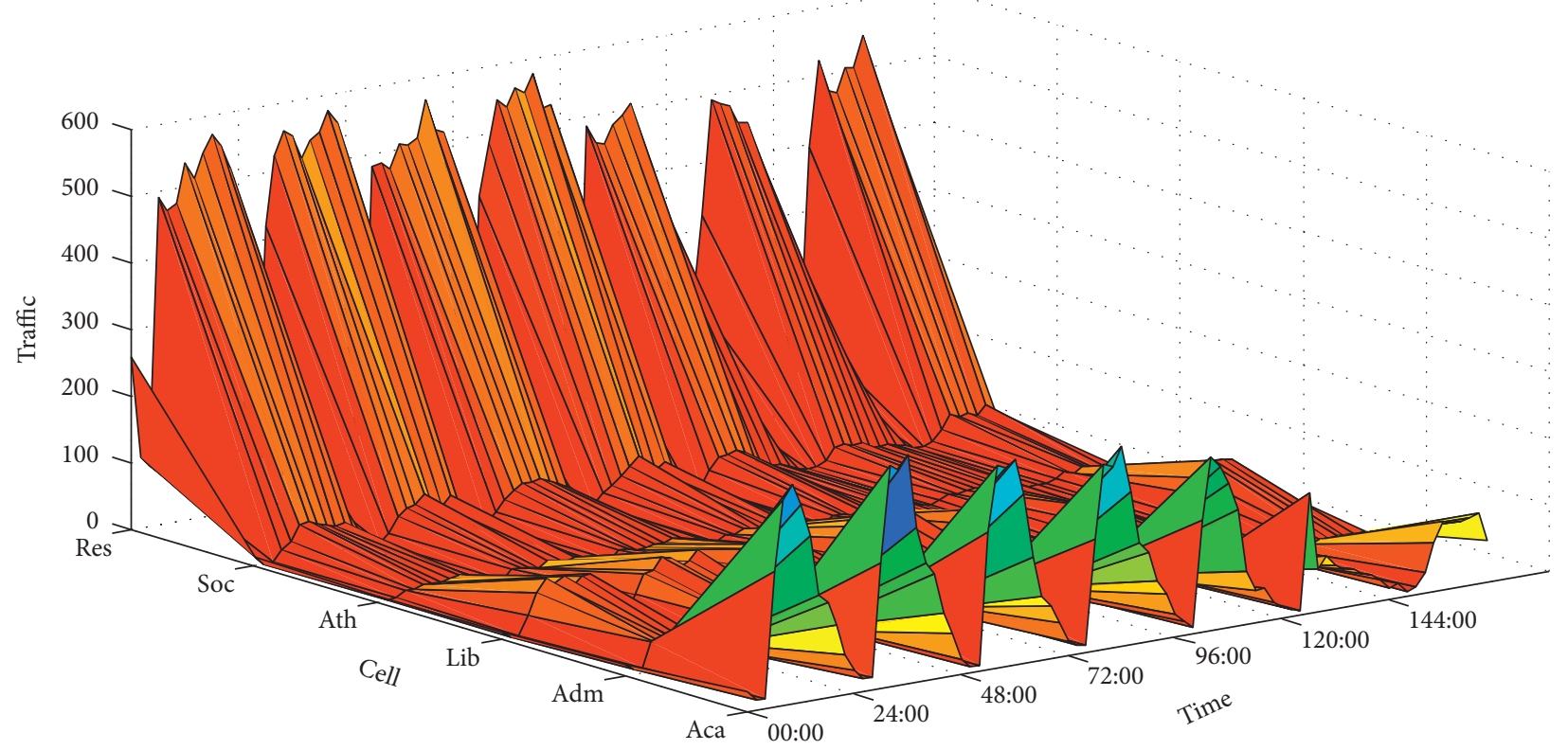

Figure 1: Temporal and spatial traffic distribution in the period of two weeks.

easily accommodate additional traffic loads. Therefore, we assume that reducing or turning off the transmitting power of part of the AP will not result in a significant increase in the call blocking probability or an increase in the jamming level.

Here, we assume that the coverage region of each AP is a hexagonal region of radius $\mathrm{R}$. We use $h_{k}^{(m)}$ to represent the channel fading index between the $m$ antennas of the $k$ AP, $\left|h_{k}^{(m)}\right|^{2}$ is a cyclically symmetric complex Gaussian random variable with zero mean and variance. The variance is shown in the following equation:

$$
\sigma_{k}^{2}=\frac{G}{d_{k}^{\alpha}}
$$

where $d_{k}$ represents the distance from the user to the $k$ th AP; $\alpha$ represents the path loss index. $G$ is the normalized gain coefficiency. Suppose $h_{k}^{(m)}$ is not related to $(k, m)$ and channel state information is available at the AP.

In determining which SA to reduce power or turn off, the optimal solution using integer programming has been proved to be a NP-Hard problem $[27,28]$. In view of the low repeat coverage of $\mathrm{Wi}-\mathrm{Fi}$ network, we adopt a method that is more effective in a specific environment: SA scaling method. Figure 2 shows one of the three scaled APs, in which we use $\beta$ to represent the proportion of the number of scaled or even closed APs to the total number of APs.

Expanding coverage of the user at the SA in the AP zoom, compensating for the loss of signal-to-noise ratio (SNR) due to increasing the distance between the AP and the user, and using cooperative communication and power control for adjacent APs, we restricted group cooperation AP to only direct adjacent AP to reduce power or turn off AP. AP scaling mode and corresponding AP cooperation mode are shown in Figure 2. In AP zoom mode, when an AP reduces power or shuts down, it releases its channel resources to adjacent active APs. Adjacent active APs then collaborate to serve the users of the scaled SA with resources obtained from the scaled AP. It can be seen from the network topology that there are only a few APs with extended coverage in multiple adjacent SAs, and the APs with extended coverage expand the transmitting power to provide user services.

Researching on users in an AP-scaled SA, we will calculate the downlink and uplink SNR, respectively. In the work [29], various off-the-shelf $\mathrm{Wi}-\mathrm{Fi}$ access points were tested to investigate customer usage patterns, and the results showed that low signal-to-noise ratios increased energy consumption of different $\mathrm{Wi}-\mathrm{Fi}$ access points by $136 \%$. The calculation of downlink and uplink SNR in the cooperative mode is as follows: (1) downlink: since the channel state information is available at the AP end, the optimal cooperative transmission mechanism is cooperative beamforming. The SNR at the receiving device is shown in the following equation:

$$
\mathrm{SNR}=\sum_{k=1, m=1}^{K, M} \frac{\left|h_{k}^{(m)}\right|^{2} P_{A P}(1-\beta)}{N_{U E}},
$$

where $P_{A P}$ is the transmission power of all APs, $N_{U E}$ is the noise power of user equipment (UE), $K$ represents the number of all APs. (2) Uplink: considering that channel state information is available at the AP, maximum ratio combining [30] is the best choice for AP cooperation. The SNR at the receiving AP is shown in in the following equation:

$$
\mathrm{SNR}=\sum_{k=1, m=1}^{K, M} \frac{P_{U E}\left|h_{k}^{(m)}\right|^{2}}{N_{k}^{(m)}},
$$

where $P_{U E}$ is the transmission power of UE; $N_{k}^{(m)}$ is the noise power of the $m$ antenna of the $k$ AP. For simplicity, it is 


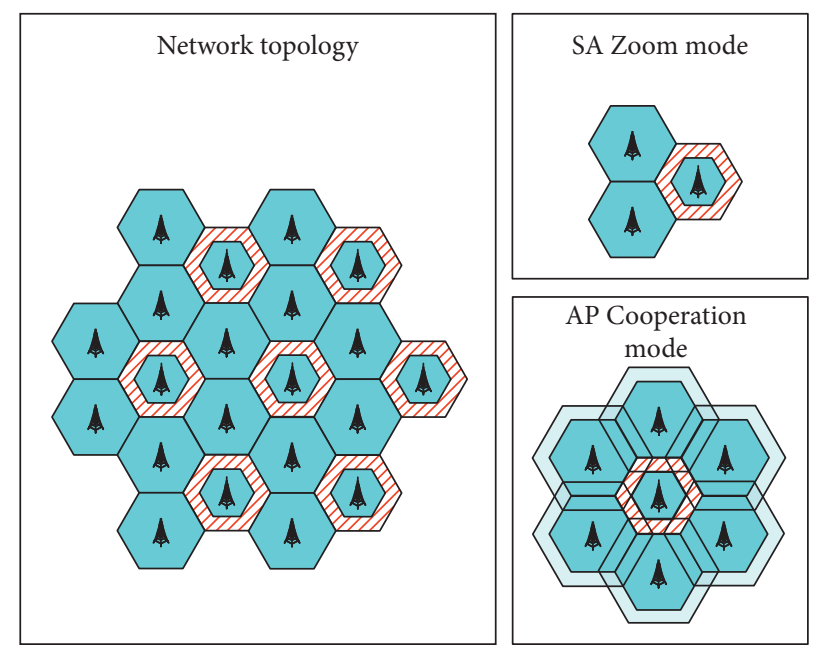

FIGURe 2: Scaling mode: scaling 1 of 3 APs.

further assumed that all $k$ and $m, N_{k}^{(m)}$, are the same, that is, $N_{k}^{(m)}=N_{A P}$, then the calculation of SNR is shown in in the following equation:

$$
\mathrm{SNR}=\frac{P_{U E}}{N_{A P}} \sum_{k=1, m=1}^{K, M}\left|h_{k}^{(m)}\right|^{2} .
$$

4.2. Outage Probability Analysis. In our work, we use the outage probability as the index to measure the user's service quality. Giving a specific SNR threshold $\gamma_{0}$, an outage is considered to occur when the instantaneous SNR is below this threshold. In order to guarantee the quality of service for all users in the SA scaled by AP, since a user may be anywhere in the SA, the average SA quality of service metric cannot measure the quality of service, we will focus on the quality of service when the users are in the worst transmission receiving location in the SA.

First, we locate the worst position in the scaling SA, given the number and location of the active APs working with it. Their geographical positions are fitted in a two-dimensional coordinate system, and a pair of coordinates are marked for $\mathrm{AP}$ and user positions, respectively. Specific coordinate basis that we assign to partner APs and users:

$$
\mathrm{AP}_{1}=\left(x_{1}, y_{1}\right), \ldots, \mathrm{AP}_{k}=\left(x_{k}, y_{k}\right) ; \quad \mathrm{UE}=(x, y) \text {. }
$$

The distance between $\mathrm{UE}$ and $\mathrm{BS}_{k}$ is

$$
d_{k}=\left[\left(x-x_{k}\right)^{2}-\left(y-y_{k}\right)^{2}\right]^{1 / 2} \text {. }
$$

After determining the worst location, we will only consider the effect of the average path loss of the decaying component:

$$
E\left[\sum_{k=1, m=1}^{K, M}\left|h_{k}^{(m)}\right|^{2}\right]=M \sum_{k=1}^{K} \frac{G}{d_{k}^{\alpha}},
$$

where $K$ represents the number of cooperative APs and $M$ represents the number of antennas of each AP. Therefore, determining the worst position in the closed hexagon region is equivalent to solving the minimization problem of the following constraint:

$$
\begin{aligned}
f(x, y)= & \sum_{k=1}^{K} \frac{1}{\left[\left(x-x_{k}\right)^{2}+\left(y-y_{k}\right)^{2}\right]^{\alpha / 2}}, \\
\text { s.t. }-\sqrt{3} R \leq & \sqrt{3} x+y \leq \sqrt{3} R \\
& -\sqrt{3} R \leq \sqrt{3} x-y \leq \sqrt{3} R \\
& -\frac{\sqrt{3}}{2} R \leq y \leq \frac{\sqrt{3}}{2} R .
\end{aligned}
$$

In the cooperation mode we proposed, the UE position of the worst case is shown in Figure 3, where the red pentagram represents the worst UE position.

Next, let us analyse the user outage probability in AP cooperation mode. Since downlink and uplink transmission scenarios have similar SNR expressions in equations (2) and (3), (9) is used uniformly in our analysis:

$$
\mathrm{SNR}=\sum_{k=1, m=1}^{K, M} \frac{P}{N}\left|h_{k}^{(m)}\right|^{2},
$$

where $N$ represents the noise power of the receiver and $P$ represents the transmitted power. On the downlink, $P=P_{A P}(1-\beta)$.

As shown in Figure 3, the distance from the worst UE position to the cooperative AP when the SA is fully scaled to close $d=\sqrt{3} R$, this geometric arrangement has the same channel gain variance $\sigma_{k}^{2}=G /(\sqrt{3} R)^{\alpha}, k=1, \ldots, K$. In cooperative mode, when SA scaling does not reach the off-state, the channel gains variance $\sigma_{k}^{2}=G /\left(\sqrt{\mu^{2}-3 \mu+3 R}\right)^{\alpha}$, where $\mu$ represents the scaling ratio of a single SA. So, equation (9) can be expressed as

$$
\mathrm{SNR}=\frac{P}{N} \frac{\sigma_{k}^{2}}{2} \sum_{k=1, m=1}^{K, M} \frac{2}{\sigma_{k}^{2}}\left|h_{k}^{(m)}\right|^{2} .
$$

Since $W=\sum_{k=1, m=1}^{K, M} 2 / \sigma_{k}^{2}\left|h_{k}^{(m)}\right|^{2}$ is a central chi-square random variable with $2 K M$ degree of freedom, the 
cumulative distribution probability of $P_{r}[W \leq \omega]$ is shown in the following equation:

$$
P_{r}[W \leq \omega]=\frac{\gamma(\mathrm{MK}, \omega / 2)}{(\mathrm{MK}-1) !}
$$

where the $\gamma$ function is $\gamma(s, x)=\int_{0}^{x} t^{s-1} e^{-t} \mathrm{~d} t$. Therefore, the outage probability can be expressed as

$$
P_{\text {out }}=P_{r}\left[\mathrm{SNR} \leq \gamma_{0}\right]=\frac{\gamma\left(\mathrm{MK},\left(\gamma_{0} N\left(\sqrt{\mu^{2}-3 \mu+3 R}\right)^{\alpha} / \mathrm{PG}\right)\right)}{(\mathrm{MK}-1) !} \text {. }
$$

In the AP cooperation mode, as shown in Figure 3, the distance between the worst position of the user and the cooperative AP after amplification constraint is $d=\sqrt{3 \mu^{2} / 4-3 \mu / 2+3 R}$. Therefore, equation (9) can be expressed as

$$
\mathrm{SNR}=\frac{P}{N} \frac{\sigma_{k}^{2}}{2} \sum_{k=1, m=1}^{K, M} \frac{2}{\sigma_{k}^{2}}\left|h_{k}^{(m)}\right|^{2},
$$

where $\sigma_{k}^{2}=G /\left(\sqrt{3 \mu^{2} / 4-3 \mu / 2+3 R}\right)^{\alpha}$. It is also a central chisquare random variable with $2 \mathrm{KM}$ degrees of freedom.

Next, we analyse the user's demand for service quality. When some APs are closed, compared with all APs, they are all active, and we need to ensure the user's minimum service quality. Therefore, we need to ensure that the user's outage probability at the scaling SA worst position can be slightly lower than or equal to the user's outage probability at the SA worst position during peak operation. Since a user is served by a single AP in an active $\mathrm{SA}$, its worst location is at the edge of the SA, as shown in the traditional pattern in Figure 3. The corresponding SNR expression equation (9) is a special case of $K=1$.

$$
\mathrm{SNR}=\frac{P}{N} \sum_{m=1}^{M}\left|h_{0}^{(m)}\right|^{2}=+\frac{P \sigma_{0}^{2}}{N 2} \sum_{m=1}^{M} \frac{2}{\sigma_{0}^{2}}\left|h_{0}^{(m)}\right|^{2},
$$

where $N$ is the noise power, $P$ is the transmission power, $\left|h_{0}^{(m)}\right|^{2}$ is the channel revenue between the user and the $m$ antenna of the AP, and its variance is $\sigma_{0}^{(m)}=G / R^{\alpha}$.

In the traditional mode without SA scaling, the same $V=$ $\sum_{m=1}^{M} 2 / \sigma_{0}^{2}\left|h_{0}^{(m)}\right|^{2}$ is a central chi-square random variable with a degree of freedom of $2 M$. Therefore, the outage probability of the traditional mode without SA scaling can be expressed as follows:

$$
P_{\text {out }}=P_{r}\left[\mathrm{SNR} \leq \frac{2 \gamma_{0} N}{P \sigma_{0}^{2}}\right]=\frac{\gamma\left(M,\left(\gamma_{0} \mathrm{NR}^{\alpha} / \mathrm{PG}\right)\right)}{(M-1) !} .
$$

4.3. Energy-Efficient Effect and Numerical Analysis. The total energy consumption $P_{\text {totle }}$ of an AP can be divided into two main parts, idle power consumption $P_{\text {idle }}$ and transmitted power consumption $P_{R F}$. $P_{\text {idle }}$ does not depend on network traffic load, that is, an active AP in the idle state does not transmit or receive. While $P_{R F}$ is dependent on network traffic load, the main is the power consumption of radio frequency (RF) output components. The transmission and reception power consumption of cooperative AP is negligible compared with that of traditional noncooperative AP transmission and reception. Therefore, it is assumed that the additional baseband signal processing power consumption is negligible.

Here, $\eta$ is defined as the ratio of $P_{R F}$ to $P_{\text {totle }}$, and $\lambda$ is defined as the ratio of the total transmission power required to the power which is required for cooperative transmission, while maintaining the same minimum quality of service level as traditional noncooperative operations. During energy-efficient operations, $\beta$ represents the percentage of all SAs scaled. The traffic load of scaled APs is shifted to other active APs, which means that $\lambda$ is increased and power saved in the network is reduced during energy-efficient operations in order to achieve the required quality of service levels. Therefore, the percentage of power saving $\xi$ during energy-efficient operation can be calculated by equation (15):

$$
\begin{aligned}
\xi & =1-\frac{\text { total consumption in energy }- \text { efficient operation }}{\text { total consumption in conventional operation }} \\
& =\frac{\beta P_{\text {total }}-\beta \lambda P_{R F}}{P_{\text {total }}} \\
& =\beta[1-\eta \lambda] .
\end{aligned}
$$

We use the proposed SA scaling strategy and AP cooperation mechanism to demonstrate some numerical analysis results and energy-efficient performance. We use the $\phi=P / \gamma_{0} \mathrm{NR}^{\alpha} / G$ function to represent the standardized SNR level of a given network based on system parameters; the $\gamma_{0} \mathrm{NR}^{\alpha} / G$ is a fixed predefined value for a given network.

Figures 4 and 5 show the outage probability curve of AP cooperation mode, where the path loss index is $\alpha=3.5$ and $\alpha=4$, respectively. Curve baseline QoS requirement indicates that there is no AP cooperation and all AP is in peak operation in the traditional mode.

From Figures 4 and 5, we can clearly see that when the outage probability is $P_{\text {out }} \in\left(10^{-4}, 10^{-3}\right)$ in the AP cooperation mode, it is better than that without the AP cooperation mode. Therefore, it can be seen that the user service quality through the AP cooperation mode can be guaranteed.

As shown in Figure 6, the proposed SA scaling mode and AP cooperation mode can significantly reduce energy consumption. Running in this energy-efficient mode can save about $35 \%$ of the energy consumption and ensure the required quality of service. It can be seen that when the path loss coefficient $\alpha=3.5$, the energy efficiency is relatively high. On the contrary, when the path loss coefficient $\alpha=4.0$, after the path loss increases, additional transmitting power is needed to ensure high service quality. It is not difficult to find from Figure 6 that the energy saving effect is relatively low. It can also be found that when the terminal probability requirement is low, the energy saving effect is relatively good. 

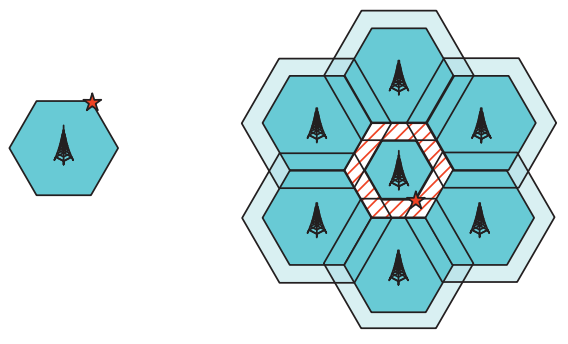

Non-cooperative mode

AP Cooperation mode

FIGURE 3: Worst case UE position.

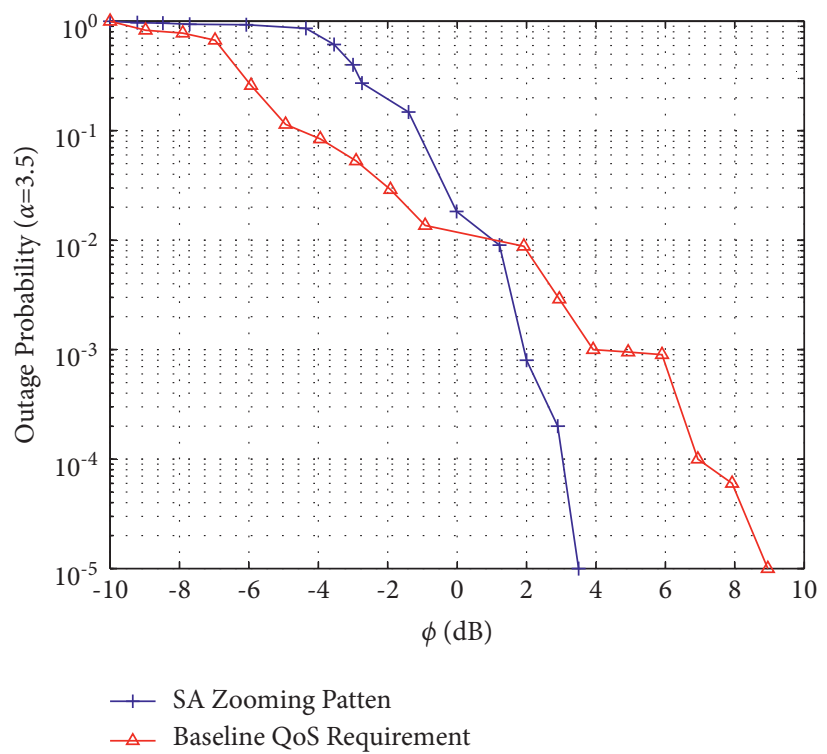

FIGURE 4: Outage probability when the path loss index of scaling mode is 3.5 .

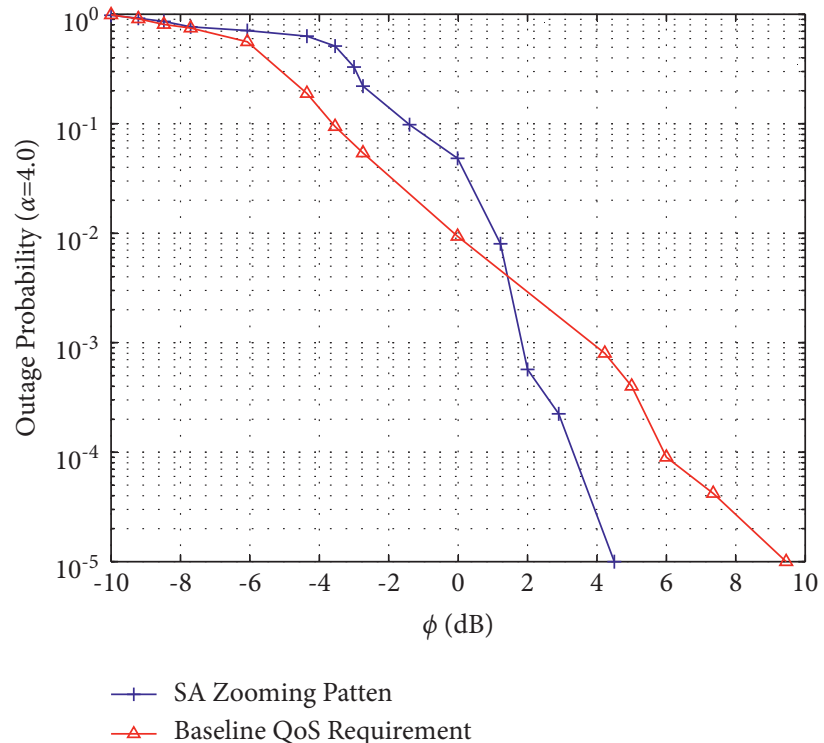

FIGURE 5: Outage probability when the path loss index of scaling mode is 4 . 


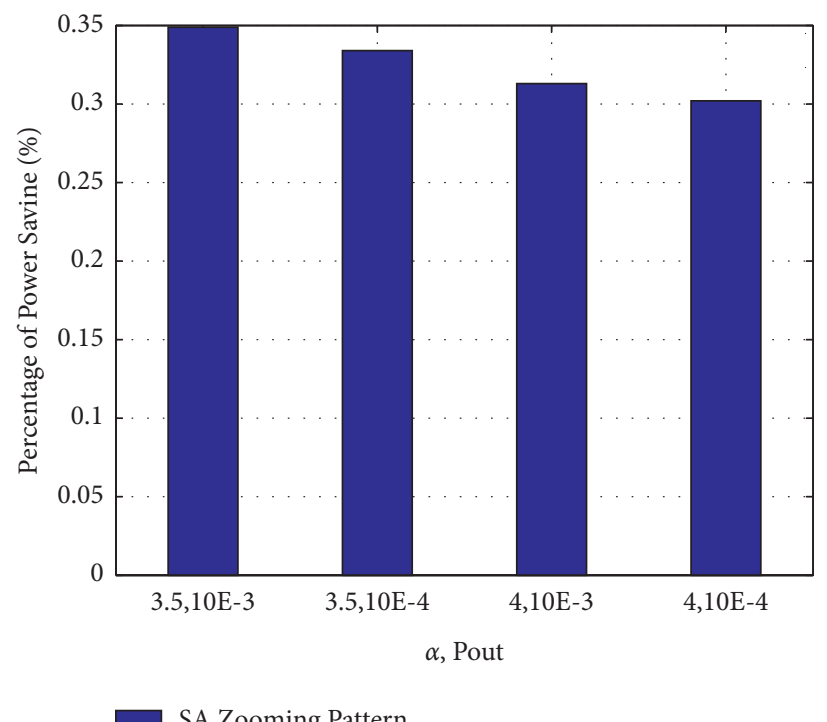

SA Zooming Pattern

Figure 6: Histogram of energy-efficient effect.

\section{Conclusion}

The energy consumption of Wi-Fi network during operation is increasing year by year. Reducing the energy consumption of Wi-Fi network has attracted extensive attention in the communication industry. Based on the real dataset, this paper analyses the spatiotemporal characteristics of the data traffic and confirms that the traffic of the Wi-Fi has tidal effect and obvious periodicity in time and space. The strategy of dynamic sleep or shutdown of AP is designed by using uneven traffic distribution to reduce the energy consumption of $\mathrm{Wi}-\mathrm{Fi}$ network. Through the simulation experiment, we find that the energy consumption can be reduced by about $35 \%$ in this mode, and the service quality can be guaranteed. In future work, we will continue to analyse larger volumes of traffic data in order to design more effective Wi-Fi energy-efficient solutions.

\section{Data Availability}

The authors used a dataset of wireless access points on the Dartmouth campus [6], where nearly 500 access points have been installed, covering most of the campus. The dataset was collected over a period of five years. The campus has 200 acres of area with more than 190 buildings, about 5,500 students and 1,200 teachers [6]. David Kotz, Tristan Henderson, Ilya Abyzov, Jihwang Yeo, CRAWDAD dataset dartmouth/campus (v. 200909-09), downloaded from https://crawdad.org/ dartmouth/campus/20090909, https://doi.org/10.15783/ C7F59T, Sep 2009.

\section{Conflicts of Interest}

The authors declare that they have no conflicts of interest.

\section{Acknowledgments}

This work was supported by the Science and Technology Project of Henan Province (Grant nos. 202102210182 and 212102210425) and Key Scientific Research Projects of Higher Education in Henan Province (Grant no. 21ZX014) in China.

\section{References}

[1] J. Lorincz, A. Capone, and M. Bogarelli, "Energy savings in wireless access networks through optimized network management," in Proceedings of the IEEE 5th International Symposium on Wireless Pervasive Computing 2010, pp. 449-454, Mondena Italy, May 2010.

[2] S. Yang, X. Yang, C. Zhang, and E. Spyrou, "Using social network theory for modeling human mobility," IEEE network, vol. 24 , no. 5 , pp. $6-13,2010$.

[3] A. S. Cacciapuoti, F. Calabrese, M. Caleffi, G. Di Lorenzo, and L. Paura, "Human-mobility enabled networks in urban environments: is there any (mobile wireless) small world out there?" Ad Hoc Networks, vol. 10, no. 8, pp. 1520-1531, 2012.

[4] M. C. González, C. A. Hidalgo, and A.-L. Barabási, "Understanding individual human mobility patterns," Nature, vol. 453, no. 7196, pp. 779-782, 2008.

[5] C. Song, Z. Qu, N. Blumm, and A.-L. Barabási, "Limits of predictability in human mobility," Science, vol. 327 , no. 5968 , pp. 1018-1021, 2010.

[6] D. Kotz, T. Henderson, I. Abyzov, and J. Yeo: CRAWDAD dataset dartmouth/campus (v.2009-09-09), down loaded from, https://crawdad.org/dartmouth/campus/20090909.

[7] Z. Li, X. Wang, and J. Zhang, "Temporal and spatial traffic analysis based on human mobility for energy efficient cellular network," KSII Transactions on Internet and Information Systems (TIIS), vol. 15, no. 1, pp. 114-130, 2021.

[8] H. Jiang, J. Li, P. Zhao, F. Zeng, Z. Xiao, and A. Iyengar, "Location privacy-preserving mechanisms in location-based 
services," ACM Computing Surveys, vol. 54, no. 1, pp. 1-36, 2021.

[9] B. Malčić, G. Gardašević, and S. Šajić, "A new algorithm for energy-efficient IEEE 802.11 access points," in Proceedings of the 2017 25th Telecommunication Forum (TELFOR), pp. 1-4, Belgrade, Serbia, November 2017.

[10] S. Sharma, S. J. Darak, and A. Srivastava, "A transfer learning framework for energy efficient Wi-Fi networks and performance analysis using real data," in Proceedings of the 2016 IEEE International Conference on Advanced Networks and Telecommunications Systems (ANTS), pp. 1-6, Bangalore, India, November 2016.

[11] X. Han, I. Samy, and L. Lazos, "Energy-efficient LTE/Wi-Fi coexistence," in Proceedings of the ICC 2020-2020 IEEE International Conference on Communications (ICC), pp. 1-7, Hoboken, NY, USA, June 2020.

[12] A. Dziedzic, V. Sathya, M. I. Rochman, M. Ghosh, and S. Krishnan, "Machine learning enabled spectrum sharing in dense LTE-U/Wi-Fi coexistence scenarios," IEEE Open Journal of Vehicular Technology, vol. 1, pp. 173-189, 2020.

[13] V. Sathya, M. Merhnoush, and M. Ghosh, "Energy detection based sensing of multiple Wi-Fi BSSs for LTE-U CSAT," in Proceedings of the 2018 IEEE Global Communications Conference (GLOBECOM), pp. 1-7, Abu Dhabi, UAE, December 2018.

[14] F. Richter, A. J. Fehske, and G. P. Fettweis, "Energy efficiency aspects of base station deployment strategies for cellular networks," in Proceedings of the 2009 IEEE 70th Vehicular Technology Conference Fall, pp. 1-5, Anchorage, September 2009.

[15] K. Son, H. Kim, Y. Yi, and B. Krishnamachari, "Base station operation and user association mechanisms for energy-delay tradeoffs in green cellular networks," IEEE Journal on Selected Areas in Communications, vol. 29, no. 8, pp. 1525-1536, 2011.

[16] Z. Kuang, G. Li, L. Zhang, H. Zhou, C. Li, and A. Liu, "Energy efficient mode selection, base station selection and resource allocation algorithm in D2D heterogeneous networks," Peerto-Peer Networking and Applications, vol. 13, no. 5, pp. 1814-1829, 2020.

[17] N. Zabetian, A. Mohammadi, and M. Masoudi, "Energy-efficient power allocation for device-to-device communications underlaid cellular networks using stochastic geometry," Transactions on Emerging Telecommunications Technologies, vol. 30, no. 12, 2019.

[18] S. Cho and W. Choi, "Energy-efficient repulsive cell activation for heterogeneous cellular networks," IEEE Journal on Selected Areas in Communications, vol. 31, no. 5, pp. 870-882, 2013.

[19] X. Weng, D. Cao, and Z. Niu, "Energy-efficient cellular network planning under insufficient cell zooming," in Proceedings of the 2011 IEEE 73rd Vehicular Technology Conference (VTC Spring), pp. 1-5, Yokohama, Japan, May, 2011.

[20] E. Oh, B. Krishnamachari, and X. Liu, "Toward dynamic energy-efficient operation of cellular network infrastructure," IEEE Communications Magazine, vol. 49, no. 6, pp. 55-61, 2011.

[21] D. Liu, Z. Cao, M. Hou, H. Rong, and H. Jiang, "Pushing the limits of transmission concurrency for low power wireless networks," ACM Transactions on Sensor Networks, vol. 16, no. 4, pp. 1-29, 2020.

[22] Y. Zhong, T. Q. S. Quek, and X. Ge, "Heterogeneous cellular networks with spatio-temporal traffic: delay analysis and scheduling," IEEE Journal on Selected Areas in Communications, vol. 35, no. 6, pp. 1373-1386, 2017.
[23] A. Lahiry, "Analytical evaluation of an antenna array system and adaptive cell densification technique for energy efficient LTE network," Wireless Personal Communications, vol. 109, no. 4, pp. 2507-2540, 2019.

[24] Y. Shuang, H. Zhao, and H. Li, "Dynamic energy allocation of commondity Wi-Fi signals using programmable coding metasurface," in Proceedings of the 2019 International Applied Computational Electromagnetics Society Symposium-China (ACES), vol. 1, pp. 1-2, Nanjing, China, August 2019.

[25] S. He, K. Xie, K. Xie, C. Xu, and J. Wang, "Interference-aware multisource transmission in multiradio and multichannel wireless network," IEEE Systems Journal, vol. 13, no. 3, pp. 2507-2518, 2019.

[26] Z. Xiao, X. Dai, and H. Jiang, "Vehicular task offloading via heat-aware MEC cooperation using game-theoretic method," IEEE Internet of Things Journal, vol. 7, no. 3, pp. 2038-2052, 2019.

[27] R. M. Karp, Reducibility Among Combinatorial Problems, Springer, Boston, MA, 1972.

[28] R. G. Michael and S. J. David, "Computers and intractability: a guide to the theory of NPcompleteness," Society for Industrial and Applied Mathematics, vol. 24, no. 1, pp. 90-91, 1979.

[29] M. Morshedi and J. Noll, "Deploying energy efficient wi-fi networks," in Proceedings of the 2019 International Conference on Wireless and Mobile Computing, Networking and Communications (WiMob), pp. 33-39, Bologna, Italy, October 2019.

[30] K. Son, E. Oh, and B. Krishnamachari, "Energy-aware hierarchical cell configuration: from deployment to operation," in Proceedings of the 2011 IEEE Conference on Computer Communications Workshops (INFOCOM WKSHPS), pp. 289-294, Shanghai, China, April 2011. 